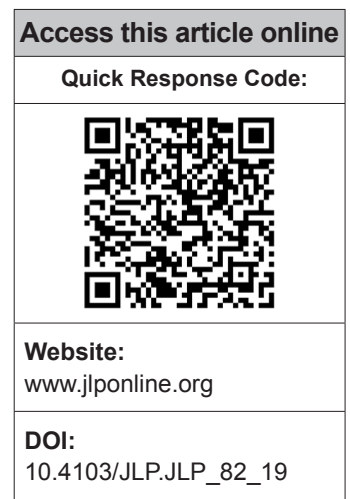

Department of Emergency Medicine, Incheon St. Mary's Hospital, College of Medicine, The Catholic University of Korea, ${ }^{1}$ Department of Nuclear Medicine, College of Medicine, Ewha Womans University, Seoul, ${ }^{2}$ Department of Emergency Medicine, College of Medicine, The Catholic University of Korea, Incheon, Korea

Address for correspondence:

Dr. Daehee Kim,

Department of Emergency

Medicine, Incheon St.

Mary's Hospital, The

Catholic University of Korea, 56 Dongsu-Ro,

Bupyeong-Gu, Incheon 21431, Korea.

E-mail:md.kim.daehee@ gmail.com

Submission: 07-05-2019 Accepted: 20-08-2019

\title{
Predictive values of
}

\section{neutrophil-lymphocyte ratio as an early indicator for severe acute pancreatitis in the emergency department patients}

\author{
Hyun Sok Park, Sang Guk In, Hai-Jeon Yoon ${ }^{1}$, Woon Jeong Lee ${ }^{2}$, Seon Hee Woo², \\ Daehee Kim
}

\begin{abstract}
:
CONTEXT: Acute pancreatitis is an acute inflammatory disease presenting with a wide range of severity.
\end{abstract}

AIMS: We investigated the predictive values of the neutrophil-lymphocyte ratio (NLR) as an indicator for severe acute pancreatitis in the emergency department patients.

SETTINGS AND DESIGN: This retrospective study was conducted on patients with acute pancreatitis who were diagnosed in the emergency department, from January 2008 to December 2017.

SUBJECTS AND METHODS: Patients were classified into either mild-to-moderate severe group or severe group according to the Revised Atlanta Classification for Acute Pancreatitis. Clinical features and laboratory blood test parameters were considered as independent variables.

STATISTICAL ANALYSIS USED: Independent variables were analyzed using the Chi-square test and Mann-Whitney U-test to determine statistically significant differences between the two groups. Logistic regression analysis and receiver operating characteristic analysis were performed to evaluate the predictive values of significantly different variables.

RESULTS: Of the 672 patients, $52(7.7 \%)$ were classified into the severe group. Tachycardia, fever, prevalence of liver cirrhosis and chronic alcoholism, white blood cell count, NLR, C-reactive protein (CRP), blood urea nitrogen (BUN), creatinine, aspartate transaminase, and total bilirubin were significantly higher in the severe group. Among them, NLR (adjusted odds ratio [aOR]: 1.13; 95\% confidence interval [Cl]: 1.081-1.181), CRP (aOR: 1.011; 95\% Cl: 1.004-1.017), BUN (aOR: 1.036; 95\% Cl: 1.004-1.069), and creatinine (aOR: 1.703; 95\% Cl: 1.008-2.877) were significant in the logistic regression analysis. NLR showed relatively high sensitivity (82.7\%) and specificity $(70 \%)$ and showed the highest area under the curve $(0.821)$.

CONCLUSIONS: The increase in NLR was associated with severe acute pancreatitis. NLR is expected to be useful as a prognostic factor in patients with acute pancreatitis who are visiting the emergency department.

Key words:

Grade approach, pancreatitis, prognosis

\section{Introduction}

Pancreatitis is an inflammatory
process of the pancreas and common

This is an open access journal, and articles are distributed under the terms of the Creative Commons Attribution-NonCommercial-ShareAlike 4.0 License, which allows others to remix, tweak, and build upon the work non-commercially, as long as appropriate credit is given and the new creations are licensed under the identical terms.

For reprints contact: reprints@medknow.com gastrointestinal cause of hospitalization. Symptoms of pancreatitis are severe abdominal pain, nausea, and vomiting. Pancreatitis is diagnosed by the presence of two out of three criteria: acute upper abdominal pain; serum amylase and/or lipase level elevation $>3$ times

How to cite this article: Park HS, In SG, Yoon HJ, Lee WJ, Woo SH, Kim D. Predictive values of neutrophil-lymphocyte ratio as an early indicator for severe acute pancreatitis in the emergency department patients. J Lab Physicians 2019;11:259-64. 
upper normal limits; and characteristic findings from an abdominal imaging study. ${ }^{[1]}$ While the overall mortality rate of acute pancreatitis is $5 \%$, this increased up to $17 \%-30 \%$ in the severe form, in contrast with $1.5 \%$ in mild pancreatitis. ${ }^{[2]}$ Advances in diagnostic and therapeutic intervention have led to a decrease in mortality, especially in severe acute pancreatitis. ${ }^{[3]}$ Therefore, early recognition of the severity of the acute pancreatitis is important and will be beneficial in identifying patients who need specific interventions or intensive care support.

There are many scoring systems which have reported including the Acute Physiology and Chronic Health Evaluation II (APACHE-II) and an obesity score, Ranson's criteria, Glasgow score, Bedside Index of Severity in Acute Pancreatitis score, harmless acute pancreatitis score, Balthazar score based on clinical and laboratory values, and computed tomography (CT) findings. ${ }^{[4-9]}$ However, none of these are sensitive or specific enough, and there has been no definitive consensus as to which scoring system should be used. In addition, Ranson's criteria and Glasgow scores take $48 \mathrm{~h}$ to complete, therefore have limitations in their ability to predict the severity of acute pancreatitis at the time of initial diagnosis and treatment.

In recent years, many serum markers have been studied for the prediction of severity of acute pancreatitis. C-reactive protein (CRP), especially interval change, has been associated with the severity of pancreatitis. ${ }^{[10]}$ Blood urea nitrogen (BUN) can be used for the early identification of pediatric patients at higher risk of developing severe acute pancreatitis. ${ }^{[11]}$ In addition, there are reports of albumin, total cholesterol, high-density lipoprotein, creatinine, and cytokines being able to predict severe acute pancreatitis. ${ }^{[12-15]}$ However, these serum markers also had their limitations and no definitive consensus as to the prognostic criteria for acute pancreatitis.

For this reason, we have focused on the neutrophil-lymphocyte ratio (NLR) as an early indicator for severe acute pancreatitis. NLR can be calculated easily from the complete blood counts and can be acquired before initial diagnosis and treatment. Several studies have reported on the predictive values of NLR in colorectal cancer, lung cancer, and pancreatic cancer. ${ }^{[16-18]}$ Recently, many studies have reported NLR is not only a cancer-specific prognostic factor but also a prognostic factor for systemic inflammatory diseases, such as bacteremia, the outcome of surgery, and acute kidney injury. ${ }^{[19-21]}$ In this study, we evaluated the predictive values of NLR as an early indicator of severe acute pancreatitis.

\section{Subjects and Methods}

\section{Study population}

The present study was conducted in the emergency department of a 1020-bed medical college-affiliated hospital located in Incheon, Republic of Korea. Incheon is a city in the Seoul metropolitan area and is the third-largest city in Korea, with a population of 2.91 million. This hospital was a tertiary referral hospital. In 2018, 60,521 people visited the emergency department of this hospital, and $9301(15.4 \%)$ were admitted, of whom $2219(3.7 \%)$ were admitted to the intensive care unit (ICU).

\section{Study design}

This was a retrospective study. The study was conducted over a period of 10 years, from January 1, 2008, to December 31, 2017. During the study, adult patients over the age of 18 years who were diagnosed with acute pancreatitis in the emergency department were included. Patients who met the following criteria were excluded: patients without contrast-enhanced abdominopelvic CT images; patients who were transferred to another hospital; patients who were self-discharged against medical advice; and patients who were admitted to another hospital with acute pancreatitis before transfer. Owing to the cross-sectional study design, we included only data from the first visit for patients who were admitted multiple times within the study period, regardless of the number of visits.

Patients were classified based on the Revised Atlanta Classification for Acute Pancreatitis. ${ }^{[22]}$ A mild grade of acute pancreatitis was classified by the absence of organ failure and the absence of local or systemic complications. Severe acute pancreatitis was classified by the presence of persistent organ failure or local complication or systemic complication. Others were classified as moderately severe acute pancreatitis. Organ failure was defined using laboratory studies and vital sign records based on the Modified Marshall Scoring System. ${ }^{[23]}$ Local complications were defined as the acute peripancreatic fluid collection, pancreatic pseudocyst, acute necrotic collection, walled-off necrosis, gastric outlet dysfunction, splenic and portal vein thrombosis, and colonic necrosis. Local complications were confirmed by the contrast-enhanced abdominopelvic CT images. A systemic complication was defined as an exacerbation of preexisting comorbidity.

The independent variables used in the analysis were age, sex, vital signs, previous medical history, and first laboratory studies after arrival at the emergency department. The previous medical history included hypertension, diabetes, liver cirrhosis, and chronic alcoholism. First laboratory studies after arrival at the 
emergency department included white blood cell (WBC) count, NLR, erythrocyte sedimentation rate (ESR), CRP, amylase, lipase, BUN, creatinine, aspartate transaminase (AST), alanine transaminase (ALT), alkaline phosphatase (ALP), and total bilirubin. The dependent variables other than the severity of acute pancreatitis included ICU admission, hospital days, local complication, cardiovascular failure, respiratory failure, renal failure, multi-organ failure, organ failure continuing $\geq 48 \mathrm{~h}$, and death.

\section{Statistical analysis}

All statistical analyses were performed using the MedCalc (version 9.5; MedCalc Software, Mariakerke, Belgium). Patients were divided into two groups (mild and moderately severe acute pancreatitis group vs. severe acute pancreatitis group). Previously collected patient data were analyzed using the Chi-square test and Mann-Whitney U-test to determine statistically significant differences between the two groups of independent variables. We performed logistic regression analyses of characteristics that significantly differed between groups to identify the independent variables associated with severe acute pancreatitis.

We evaluated the predictive performance of each serum biomarker that was significant in logistic regression analysis using receiver operating characteristic (ROC) analysis. The area under the curve (AUC) of the serum biomarker was compared with each other for predicting severe acute pancreatitis. The sensitivity and specificity of acute severe pancreatitis were calculated by the optimal cutoff value. Statistical significance was defined when the $P<0.05$.

\section{Ethics statement}

The data collected were used for study purposes only. This study was approved by the Institutional Review Board of the Catholic University of Korea Catholic Medical Center, where the study was conducted. The approval number was OC18RESI0079.

\section{Results}

A total of 702 patients were diagnosed with acute pancreatitis and admitted through the emergency department within the study from January 1, 2008, to December 31, 2017. Of these, a total of 672 patients were enrolled, except for seven patients without contrast-enhanced abdominopelvic CT images, two patients who were admitted to another hospital with acute pancreatitis before the transfer, and 21 patients who were self-discharged against medical advice. No patient was excluded because of transfer to another hospital or missing data. A total of 672 patients were classified into three grades according to the Atlanta classification.
Four hundred and seventy-nine patients $(71.3 \%)$ were classified to mild grade, 141 patients $(21 \%)$ were classified to moderately severe grade, and 52 patients $(7.7 \%)$ were classified to severe grade.

Clinical characteristics of patients with acute pancreatitis by grading of severity were as follows [Table 1]. Systolic and diastolic blood pressures were significantly higher in the severe group than in the mild and moderately severe group. Furthermore, heart rate and body temperature were significantly higher in the severe group than in the mild and moderately severe group. The severe group was more likely to have liver cirrhosis and chronic alcoholism than the mild and moderately severe group. Other factors did not significantly differ between groups. Laboratory studies of patients with acute pancreatitis by grading of severity were as follows [Table 2]. WBC count, NLR, $\mathrm{CRP}, \mathrm{BUN}$, creatinine, AST, and total bilirubin were significantly higher in the severe group than in the mild and moderately severe group. ESR, amylase, lipase, ALT, and ALP did not significantly differ between groups.

The characteristics related to prognostic factors were as follows [Table 3]. The proportion of ICU admission and the duration of hospital days differed significantly between the mild and moderately severe group and the severe group. The severe group was more likely to have organ failure than the mild and moderately severe group. Multi-organ failure, organ failure continuing $48 \mathrm{~h}$, and death were observed only in the severe group.

The results of the logistic regression analysis of factors shown to be as an early indicator for severe acute

Table 1: Comparison of the clinical characteristics between the mild and moderately severe group and severe group

\begin{tabular}{|c|c|c|c|}
\hline & $\begin{array}{l}\text { Mild and moderately } \\
\text { severe }(n=620)\end{array}$ & Severe $(n=52)$ & $P$ \\
\hline Age (years) & $51(40-62)$ & $54(47-64)$ & 0.173 \\
\hline Male & $408(65.8)$ & $36(69.2)$ & 0.616 \\
\hline $\begin{array}{l}\text { Systolic blood } \\
\text { pressure }(\mathrm{mmHg})\end{array}$ & $132.0(117.0-149.0)$ & $110.5(87.0-126.8)$ & $<0.001$ \\
\hline $\begin{array}{l}\text { Diastolic blood } \\
\text { pressure }(\mathrm{mmHg})\end{array}$ & $82.0(69.0-91.8)$ & $67.5(56.3-77.8)$ & $<0.001$ \\
\hline Heart rate (/min) & $88.0(74.0-103.8)$ & $102.0(86.0-120.8)$ & $<0.001$ \\
\hline $\begin{array}{l}\text { Respiratory rate } \\
\text { (/min) }\end{array}$ & $20.0(18.0-20.0)$ & $20.0(18.0-20.0)$ & 0.698 \\
\hline $\begin{array}{l}\text { Body temperature } \\
\left({ }^{\circ} \mathrm{C}\right)\end{array}$ & $36.4(36.0-36.8)$ & $36.6(36.2-37.1)$ & 0.022 \\
\hline \multicolumn{4}{|l|}{$\begin{array}{l}\text { Previous medical } \\
\text { history }\end{array}$} \\
\hline Hypertension & $144(23.2)$ & $11(21.2)$ & 0.733 \\
\hline Diabetes & $123(19.8)$ & $13(25.0)$ & 0.374 \\
\hline Liver cirrhosis & $53(8.5)$ & $10(19.2)$ & 0.022 \\
\hline $\begin{array}{l}\text { Chronic } \\
\text { alcoholism }\end{array}$ & $236(38.1)$ & $28(53.8)$ & 0.025 \\
\hline
\end{tabular}

Values are expressed as $n(\%)$ or median (IQR). IQR=Interquartile range 
pancreatitis are presented in Table 4. Among the serum markers, NLR, CRP, BUN, creatinine, and WBC count were independent prognostic factors for severe acute pancreatitis. Among clinical characteristics, systolic blood pressure and heart rate were independent prognostic factors.

Serum markers with an AUC significantly different from 0.5 were NLR, CRP, creatinine, and WBC count. The sensitivity and specificity of the prognostic variables with a significant AUC for severe acute pancreatitis are listed in Table 5. Among serum markers, NLR showed a relatively high sensitivity of $82.7 \%$ and relatively high specificity of $70 \%$. According to the ROC comparison

Table 2: Comparison of first laboratory studies after arrival at the emergency department between the mild and moderately severe group and severe group

\begin{tabular}{|c|c|c|c|}
\hline & $\begin{array}{l}\text { Mild and moderately } \\
\text { severe }(n=620)\end{array}$ & Severe $(n=52)$ & $P$ \\
\hline \multicolumn{4}{|l|}{ WBC } \\
\hline $\begin{array}{l}\text { Count } \\
\left(10^{3} / \mu \mathrm{L}\right)\end{array}$ & $10.90(8.15-13.63)$ & $12.61(8.71-19.05)$ & 0.018 \\
\hline NLR & $5.70(3.02-9.94)$ & $14.85(9.18-24.01)$ & $<0.001$ \\
\hline ESR & $12.00(5.00-35.00)$ & $17.50(4.25-47.75)$ & 0.563 \\
\hline CRP (mg/L) & $0.80(0.70-1.00)$ & $71.1(18.58-149.01)$ & $<0.001$ \\
\hline $\begin{array}{l}\text { Amylase } \\
\text { (IU/L) }\end{array}$ & $\begin{array}{c}336.50 \\
(112.00-935.50)\end{array}$ & $\begin{array}{c}462.50 \\
(113.00-1213.25)\end{array}$ & 0.662 \\
\hline Lipase (U/L) & $\begin{array}{c}655.60 \\
(112.00-935.50)\end{array}$ & $\begin{array}{c}423.80 \\
(84.45-2068.68)\end{array}$ & 0.156 \\
\hline BUN (mg/dL) & $13.90(10.60-18.08)$ & $22.20(12.80-36.70)$ & $<0.001$ \\
\hline $\begin{array}{l}\text { Creatinine } \\
(\mathrm{mg} / \mathrm{dL})\end{array}$ & $0.80(0.70-1.00)$ & $1.30(0.80-2.58)$ & $<0.001$ \\
\hline AST (U/L) & $54.00(26.00-188.00)$ & $92.00(33.00-303.25)$ & 0.039 \\
\hline ALT (U/L) & $44.50(21.00-110.00)$ & $41.00(16.50-128.25)$ & 0.848 \\
\hline ALP (U/L) & $123.00(80.00-225.75)$ & $132.50(88.00-222.00)$ & 0.824 \\
\hline $\begin{array}{l}\text { Total bilirubin } \\
\text { (mg/dL) }\end{array}$ & $1.10(0.70-1.90)$ & $1.55(0.90-2.65)$ & 0.002 \\
\hline
\end{tabular}

Values are expressed as median (IQR). IQR=Interquartile range, WBC=White blood cell, ESR=Erythrocyte sedimentation rate, $\mathrm{CRP}=\mathrm{C}$-reactive protein,

BUN=Blood urea nitrogen, $A S T=A$ spartate transaminase, $A L T=A l a n i n e$

transaminase, $A L P=A l k a l i n e$ phosphatase, NLR=Neutrophil-lymphocyte ratio

Table 3: Comparison of the prognostic factors between the mild and moderately severe group and severe group

\begin{tabular}{lccc}
\hline & $\begin{array}{c}\text { Mild and moderately } \\
\text { severe }(n=620)\end{array}$ & $\begin{array}{c}\text { Severe } \\
(n=52)\end{array}$ & $P$ \\
\hline ICU admission & $35(5.6)$ & $23(44.2)$ & $<0.001$ \\
Hospital days & $7(5.0-10.0)$ & $12(6.0-21.8)$ & $<0.001$ \\
Local complication & $132(21.3)$ & $37(71.2)$ & 0.206 \\
Organ failure & & & \\
$\quad$ Cardiovascular failure & $5(0.8)$ & $21(40.4)$ & $<0.001$ \\
Respiratory failure & 0 & $29(55.8)$ & $<0.001$ \\
Renal failure & 0 & $29(55.8)$ & $<0.001$ \\
$\quad$ Multi-organ failure & 0 & $24(46.2)$ & $<0.001$ \\
Organ failure $>48 \mathrm{~h}$ & 0 & $52(100.0)$ & $<0.001$ \\
Expired & 0 & $4(7.7)$ & $<0.001$ \\
\hline
\end{tabular}

Values are expressed as $n(\%)$ or median (IQR). ICU=Intensive care unit, $\mathrm{IQR}=$ Interquartile range analysis, the AUC of NLR was higher than other serum markers. The ROC results of serum markers are shown in Figure 1.

\section{Discussion}

We observed that increases in NLR, CRP, BUN, creatinine, and WBC count were associated with severe acute pancreatitis. Furthermore, we observed that

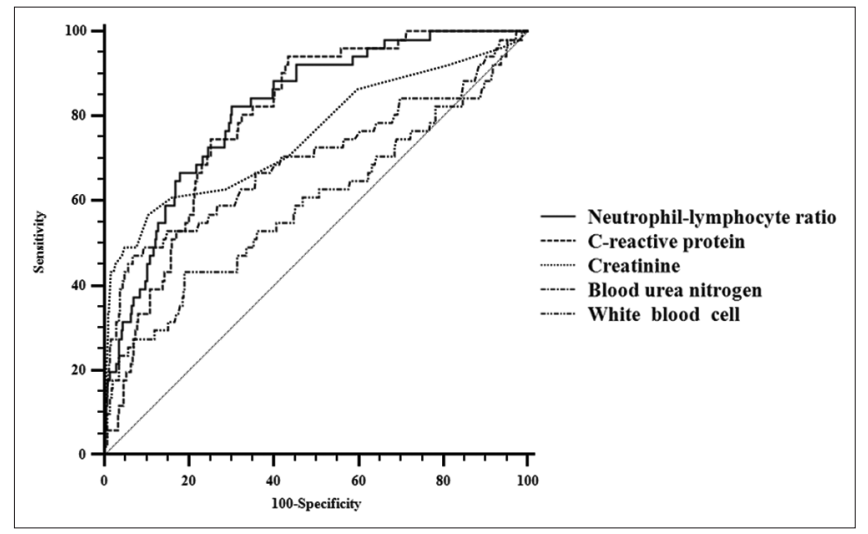

Figure 1: Receiver operating characteristic curve of serum biomarkers for distinguishing severe acute pancreatitis. These results suggest that neutrophil-lymphocyte ratio has high predictive values as an early indicator for severe acute pancreatitis

Table 4 Results of logistic regression analysis

\begin{tabular}{lcc}
\hline & aOR $(95 \% \mathrm{Cl})$ & $\boldsymbol{P}$ \\
\hline NLR & $1.143(1.091-1.197)$ & $<0.001$ \\
CRP (mg/L) & $1.011(1.004-1.017)$ & 0.001 \\
Systolic blood pressure $(\mathrm{mmHg})$ & $0.966(0.94-0.994)$ & 0.017 \\
BUN (mg/dL) & $1.036(1.004-1.069)$ & 0.029 \\
Heart rate $(/ \mathrm{min})$ & $1.023(1.001-1.047)$ & 0.043 \\
WBC count $\left(10^{3} / \mathrm{\mu L}\right)$ & $1.094(1.002-1.196)$ & 0.046 \\
Creatinine $(\mathrm{mg} / \mathrm{dL})$ & $1.703(1.008-2.877)$ & 0.046 \\
Diastolic blood pressure $(\mathrm{mmHg})$ & $0.998(0.959-1.038)$ & 0.905 \\
Body temperature $\left({ }^{\circ} \mathrm{C}\right)$ & $1.115(0.676-1.84)$ & 0.671 \\
Liver cirrhosis & $1.858(0.554-6.236)$ & 0.316 \\
Chronic alcoholics & $1.688(0.708-4.023)$ & 0.237 \\
AST $(\mathrm{U} / \mathrm{L})$ & $1(0.999-1.001)$ & 0.712 \\
Total bilirubin $(\mathrm{mg} / \mathrm{dL})$ & $0.925(0.76-1.126)$ & 0.438 \\
\hline
\end{tabular}

$\mathrm{Cl}=$ Confidence interval, aOR=Adjusted odds ratio,

NLR=Neutrophil-lymphocyte ratio, $\mathrm{CRP}=\mathrm{C}$-reactive protein, $\mathrm{BUN}=\mathrm{Blood}$ urea nitrogen, $\mathrm{AST}=\mathrm{Aspartate}$ transaminase, $\mathrm{WBC}=$ White blood cell

Table 5: Predictive performance of serum markers as an early indicator for severe acute pancreatitis; the results from receiver operating characteristic analysis

\begin{tabular}{lccccc}
\hline Variables & Cutoff & AUC & $\boldsymbol{P}$ & $\begin{array}{c}\text { Sensitivity } \\
(\%)\end{array}$ & $\begin{array}{c}\text { Specificity } \\
(\%)\end{array}$ \\
\hline NLR & 8.59 & 0.821 & $<0.001$ & 82.7 & 70.0 \\
CRP $(\mathrm{mg} / \mathrm{L})$ & 7.22 & 0.796 & $<0.001$ & 94.1 & 56.5 \\
Creatinine $(\mathrm{mg} / \mathrm{dL})$ & 1.10 & 0.759 & $<0.001$ & 57.7 & 89.5 \\
BUN $(\mathrm{mg} / \mathrm{dL})$ & 26.00 & 0.705 & $<0.001$ & 48.1 & 93.2 \\
WBC $\left(10^{3} / \mu \mathrm{L}\right)$ & 14.54 & 0.599 & 0.039 & 44.2 & 81.1 \\
\hline
\end{tabular}

$\mathrm{AUC}=$ The area under the curve, NLR=Neutrophil-lymphocyte ratio,

$\mathrm{CRP}=\mathrm{C}$-reactive protein, $\mathrm{BUN}=\mathrm{Blood}$ urea nitrogen, $\mathrm{WBC}=$ White blood cell 
changes in some vital signs (systolic blood pressure and heart rate) were associated with severe acute pancreatitis. Among them, NLR showed the highest association with severe acute pancreatitis. This suggests that NLR can be an early indicator to predict severe acute pancreatitis.

WBC count is widely used as a biomarker of infection and inflammation. WBC count is included in some scoring systems that predict the prognosis of acute pancreatitis such as APACHE-II and Ranson's criteria. ${ }^{[4,5]}$ NLR is a value obtained from the differential count of the WBC counts previously used. Therefore, further investigation is not necessary, and it can be applied to the management of acute pancreatitis easily.

There were several previous studies that analyzed the association between NLR and prognosis of acute pancreatitis. In 2011, Azab et al. analyzed the association between NLR, ICU admission, and length of stay in the hospital and suggested that NLR can be usefully used as a prognostic predictor of acute pancreatitis. ${ }^{[24]}$ In 2017, as in this study, Jeon and Park suggested that the increase in NLR was associated with the severity classification of acute pancreatitis performed according to the Atlanta Classification for Acute Pancreatitis. ${ }^{[25]}$ However, the above research was a study comparing NLR between the mild and moderately severe group to the severe group. It was not a study that analyzed the association between NLR and severe acute pancreatitis, and a regression analysis was not performed with other serum biomarkers. As a result, there were very few references using NLR for the prediction of severe acute pancreatitis.

In this study, among patients who were diagnosed and admitted with acute pancreatitis, the proportion of severe grade of pancreatitis was $7.7 \%$. This result is not significantly different from the $7 \%$ reported in the previous study conducted in the department of internal medicine of a tertiary referral hospital. ${ }^{[26]}$ This indicates that most patients with severe acute pancreatitis are admitted through the emergency department. Therefore, early prediction of severe acute pancreatitis in the emergency department can be clinically important and may benefit the prognosis of the patients.

Among clinical characteristics, systolic blood pressure, diastolic blood pressure, heart rate, and body temperature were significantly different between the two groups. These results were consistent with those of previous studies, which indicated that abnormal vital signs were associated with a higher risk of severe acute pancreatitis. ${ }^{[4,27]}$ However, vital signs fluctuate and are difficult to generalize, there is a limitation in using only vital signs as an early indicator for severe acute pancreatitis in the emergency department patients.
Among serum biomarkers, WBC count, NLR, CRP, BUN, creatinine, AST, and total bilirubin were significantly higher in the severe group. These results were consistent with those of previous studies showing that WBC count, NLR, CRP, BUN, and creatinine were associated with the severity of acute pancreatitis. ${ }^{[4,5,10,11,15,24]}$ The above serum biomarkers showed a significant difference also in the logistic regression analysis of this study. In contrast, previous studies related to AST and total bilirubin were not found. In addition, AST and total bilirubin were not significantly different in the logistic regression analysis of this study. This difference is expected to be a result caused by confounders.

In the logistic regression analysis, WBC count, NLR, $\mathrm{CRP}, \mathrm{BUN}$, and creatinine were significant variables. Among significant variables, the odds ratios of NLR were the highest, and $P$ value of NLR was the lowest. In the ROC analysis, using the variables that were significant in the logistic regression analysis, NLR exhibited more desirable results than other serum biomarkers. Compared to other serum biomarkers, both sensitivity and specificity were relatively high, and AUC was the highest. These results suggest that NLR has high predictive values as an early indicator for severe acute pancreatitis in the emergency department patients.

This study had several limitations. First, it is possible that small populations of patients with severe pancreatitis in this study have caused statistical errors. Due to the low prevalence of severe pancreatitis, the number of patients with severe pancreatitis was only 52, despite having collected data for 10 years. Therefore, to evaluate more accurate predictive values of NLR for severe pancreatitis, a prospective multi-center trial study should be performed additionally. Second, as the data were collected from the emergency department of a tertiary referral hospital, it is possible that progression to severe acute pancreatitis has been underestimated, and this possibility may have affected the results of this study. Third, the analysis of the prognosis of severe acute pancreatitis was not sufficient. For some serum biomarkers to be clinically easy to use for the management of acute pancreatitis, the biomarker must be able to predict the prognosis of acute pancreatitis. However, since the proportion of patients with poor prognosis represented by ICU admission, permanent disability, and expire had been low, we had to perform the study of indicator for the prediction of the severe grade of acute pancreatitis. Finally, this was a retrospective study based on medical records. In retrospective studies, experimental design and statistical analysis cannot always completely control for confounders.

\section{Conclusions}

Increased NLR was associated with severe acute 
pancreatitis. We would like to suggest that NLR used as a prognostic biomarker in patients admitted to the emergency department with acute pancreatitis.

\section{Financial support and sponsorship}

This research was supported by a Grant of Translational $\mathrm{R}$ and $\mathrm{D}$ Project through the Institute for Bio-Medical convergence, Incheon St. Mary's Hospital, The Catholic University of Korea (IBC 2019-13).

\section{Conflicts of interest}

There are no conflicts of interest.

\section{References}

1. Nesvaderani M, Eslick GD, Cox MR. Acute pancreatitis: Update on management. Med J Aust 2015;202:420-3.

2. Cavallini G, Frulloni L, Bassi C, Gabbrielli A, Castoldi L, Costamagna $G$, et al. Prospective multicentre survey on acute pancreatitis in Italy (ProInf-AISP): Results on 1005 patients. Dig Liver Dis 2004;36:205-11.

3. Mutinga M, Rosenbluth A, Tenner SM, Odze RR, Sica GT, Banks PA. Does mortality occur early or late in acute pancreatitis? Int J Pancreatol 2000;28:91-5.

4. Johnson CD, Toh SK, Campbell MJ. Combination of APACHE-II score and an obesity score (APACHE-O) for the prediction of severe acute pancreatitis. Pancreatology 2004;4:1-6.

5. Ranson JH, Rifkind KM, Turner JW. Prognostic signs and nonoperative peritoneal lavage in acute pancreatitis. Surg Gynecol Obstet 1976;143:209-19.

6. Blamey SL, Imrie CW, O'Neill J, Gilmour WH, Carter DC. Prognostic factors in acute pancreatitis. Gut 1984;25:1340-6.

7. Park JY, Jeon TJ, Ha TH, Hwang JT, Sinn DH, Oh TH, et al. Bedside index for severity in acute pancreatitis: Comparison with other scoring systems in predicting severity and organ failure. Hepatobiliary Pancreat Dis Int 2013;12:645-50.

8. Lankisch PG, Weber-Dany B, Hebel K, Maisonneuve P, Lowenfels AB. The harmless acute pancreatitis score: A clinical algorithm for rapid initial stratification of nonsevere disease. Clin Gastroenterol Hepatol 2009;7:702-5.

9. Balthazar EJ, Robinson DL, Megibow AJ, Ranson JH. Acute pancreatitis: Value of $\mathrm{CT}$ in establishing prognosis. Radiology 1990;174:331-6.

10. Stirling AD, Moran NR, Kelly ME, Ridgway PF, Conlon KC. The predictive value of C-reactive protein (CRP) in acute pancreatitis - Is interval change in CRP an additional indicator of severity? HPB (Oxford) 2017;19:874-80.

11. Vitale DS, Hornung L, Lin TK, Nathan JD, Prasad S, Thompson T, et al. Blood urea nitrogen elevation is a marker for pediatric severe acute pancreatitis. Pancreas 2019;48:363-6.

12. Hong W, Lin S, Zippi M, Geng W, Stock S, Basharat Z, et al. Serum albumin is independently associated with persistent organ failure in acute pancreatitis. Can J Gastroenterol Hepatol 2017;2017:5297143.
13. Hong $\mathrm{W}$, Zimmer $\mathrm{V}$, Basharat Z, Zippi M, Stock S, Geng W, et al. Association of total cholesterol with severe acute pancreatitis: A U-shaped relationship. Clin Nutr 2019. pii: S0261-5614 (19) 30041-X.

14. Hong W, Lin S, Zippi M, Geng W, Stock S, Zimmer V, et al. High-density lipoprotein cholesterol, blood urea nitrogen, and serum creatinine can predict severe acute pancreatitis. Biomed Res Int 2017;2017:1648385.

15. Deng LH, Hu C, Cai WH, Chen WW, Zhang XX, Shi N, et al. Plasma cytokines can help to identify the development of severe acute pancreatitis on admission. Medicine (Baltimore) 2017;96:e7312.

16. Walsh SR, Cook EJ, Goulder F, Justin TA, Keeling NJ. Neutrophil-lymphocyte ratio as a prognostic factor in colorectal cancer. J Surg Oncol 2005;91:181-4.

17. Sarraf KM, Belcher E, Raevsky E, Nicholson AG, Goldstraw P, Lim E. Neutrophil/lymphocyte ratio and its association with survival after complete resection in non-small cell lung cancer. J Thorac Cardiovasc Surg 2009;137:425-8.

18. Stotz M, Gerger A, Eisner F, Szkandera J, Loibner H, Ress AL, et al. Increased neutrophil-lymphocyte ratio is a poor prognostic factor in patients with primary operable and inoperable pancreatic cancer. Br J Cancer 2013;109:416-21.

19. de Jager CP, van Wijk PT, Mathoera RB, de Jongh-Leuvenink J, van der Poll T, Wever PC. Lymphocytopenia and neutrophil-lymphocyte count ratio predict bacteremia better than conventional infection markers in an emergency care unit. Crit Care 2010;14:R192.

20. Gibson PH, Croal BL, Cuthbertson BH, Small GR, Ifezulike AI, Gibson G, et al. Preoperative neutrophil-lymphocyte ratio and outcome from coronary artery bypass grafting. Am Heart J 2007;154:995-1002.

21. Yilmaz H, Cakmak M, Inan O, Darcin T, Akcay A. Can neutrophil-lymphocyte ratio be independent risk factor for predicting acute kidney injury in patients with severe sepsis? Ren Fail 2015;37:225-9.

22. Banks PA, Bollen TL, Dervenis C, Gooszen HG, Johnson CD, Sarr MG, et al. Classification of acute pancreatitis--2012: Revision of the Atlanta classification and definitions by international consensus. Gut 2013;62:102-11.

23. Marshall JC, Cook DJ, Christou NV, Bernard GR, Sprung CL, Sibbald WJ. Multiple organ dysfunction score: A reliable descriptor of a complex clinical outcome. Crit Care Med 1995;23:1638-52.

24. Azab B, Jaglall N, Atallah JP, Lamet A, Raja-Surya V, Farah B, et al. Neutrophil-lymphocyte ratio as a predictor of adverse outcomes of acute pancreatitis. Pancreatology 2011;11:445-52.

25. Jeon TJ, Park JY. Clinical significance of the neutrophil-lymphocyte ratio as an early predictive marker for adverse outcomes in patients with acute pancreatitis. World J Gastroenterol 2017;23:3883-9.

26. Huh JH, Kim JW, Lee KJ. Vitamin D deficiency predicts severe acute pancreatitis. United European Gastroenterol J 2019;7:90-5.

27. Singh VK, Wu BU, Bollen TL, Repas K, Maurer R, Mortele KJ, et al. Early systemic inflammatory response syndrome is associated with severe acute pancreatitis. Clin Gastroenterol Hepatol 2009;7:1247-51. 